\title{
Target-oriented time-lapse waveform inversion using redatumed data: Feasibility and robustness \\ Yuanyuan Li ${ }^{*}$, Qiang Guo, Tariq Alkhalifah and Vladimir Kazei \\ King Abdullah University of Science and Technology (KAUST)
}

\section{Summary}

Seismic monitoring of the changes in the subsurface induced by various types of injections into reservoirs is important, yet challenging. Time-lapse waveform inversion can retrieve quantitative estimates of subsurface property changes. Considering that property changes usually occur in a limited region rather than the whole subsurface, we present a target-oriented time-lapse waveform inversion method, which allows for dynamic monitoring of the target of interest. We employ a redatuming technique to produce virtual data at a desired datum level for the target-oriented inversion. Given the redatumed time-lapse data, the property changes can be quantitatively estimated from the data difference for the virtual survey using a doubledifference waveform inversion (DDWI). In the numerical examples, the dependence of the inversion performance on the quality of overburden model and its robustness to nonrepeatable acquisition survey and random noise is investigated. The numerical results demonstrate that the inversion method is capable of recovering the time-lapse changes reasonably well under some challenging circumstances. We will show field data examples at the conference.

\section{Introduction}

Time-lapse seismic data are widely used to monitor the property changes in reservoir caused by fluid injection and hydrocarbon production. The dynamic monitoring of these changes facilitates evaluating the related production activities and designing production plans (Johnston, 2013).

Full waveform inversion (FWI) has emerged as a promising technique for retrieving subsurface properties by solving a data-driven optimization problem iteratively (Tarantola, 1984; Vigh et al., 2014; Choi and Alkhalifah, 2011; Li et al., 2018; Li and Alkhalifah, 2019; Song and Alkhalifah, 2020). Time-lapse FWI is a straightforward extension of FWI to time-lapse seismic data and has gained considerable interest (Hicks et al., 2016; Kazei and Alkhalifah, 2018). Despite that the time-lapse FWI technique inherits the high resolution of conventional FWI, the computational burden for practical scale applications becomes even more intense because more FWI runs are involved (Yuan et al., 2017; Li et al., 2020). Considering that the property changes usually occur in a limited area rather than the whole region, the computational cost can be reduced using a target-oriented strategy, which allows inverting for a localized model
(Ayeni and Biondi, 2010; Borisov and Singh, 2015). The redatuming technique enables retrieving new Green's functions and generating virtual shot gathers as if the sources and receivers were placed at a desired datum level. In this case, the redatumed data can be the focus of a targetoriented inversion. Guo and Alkhalifah (2019b) show the advantages of the localized inversion using the redatumed data.

The target-oriented concept has shown its promise in some FWI applications (van Manen et al., 2007; Yang et al., 2012; Yuan et al., 2017; Vasconcelos et al., 2017). Yang et al. (2012) performed target-oriented time-lapse waveform inversion using virtual survey data, which admits more efficiency and faster convergence compared to standard FWI method. Yuan et al. (2017) applied the target-oriented concept to time-lapse elastic waveform inversion by combining source-side wavefield injection and receiverside extrapolation method. The redatuming is expected to improve time-lapse repeatability by suppressing 4D unrepeatable noise such as that induced by near-surface diurnal and seasonal weather cycles as well as small changes in acquisition geometry and shot coupling (Bakulin and Calvert, 2006).

In this work, we enhance the time-lapse inversion application with a waveform redatuming technique that transforms our surface recorded data to data recorded at a datum right above the zone of interest using a datuming based inversion. The redatuming operator is described by least square-based extended image, whose extended dimensions involve space and time (Guo and Alkhalifah, 2019a). With the redatumed time-lapse data, we apply a double-difference waveform inversion (DDWI) method, proposed by Denli and Huang (2009), to infer the timelapse model changes in a target region. We investigate the feasibility and robustness of the proposed inversion algorithm using numerical tests, with analyses focused on the quality of the overburden model and time-lapse survey repeatability.

\section{Theory}

We consider that a Green's function, G, obeys the following acoustic wave equation:

$$
\left(\frac{1}{v^{2}} \frac{\partial^{2}}{\partial t^{2}}-\nabla^{2}\right) \mathrm{G}\left(\mathbf{x}, \mathbf{x}_{s}, t\right)=\delta\left(\mathbf{x}-\mathbf{x}_{s}, t\right),
$$

where, $\mathbf{x}_{s}$ is the source location, $v$ is the medium velocity, $\mathbf{x}$ and $t$ are space coordinates and time. To simplify later 


\section{Target-oriented time-lapse waveform inversion: Feasibility and robustness}

derivations, the modeling operator can be shortened as $\mathbf{F}=\frac{1}{v^{2}} \frac{\partial^{2}}{\partial t^{2}}-\nabla^{2}$.

The upgoing Green's function $\mathrm{G}^{-}$at a virtual receiver $\mathbf{x}_{v r}$, generated by a surface source at $\mathbf{x}_{s}$, can be written as a convolution-type representation (Wapenaar and van der Neut, 2010):

$$
\mathrm{G}^{-}\left(\mathbf{x}_{v r}, \mathbf{x}_{s}, t\right)=\int_{S_{D}} \mathrm{~V}\left(\mathbf{x}_{v r}, \mathbf{x}_{v s}, t\right) * \mathrm{G}^{+}\left(\mathbf{x}_{v s}, \mathbf{x}_{s}, t\right) d \mathbf{x}_{v s},
$$

where, $*$ refers to a convolution operator, $\mathrm{G}^{+}$represents the downgoing Green's function and $\mathrm{V}$ represents the Green's function for the virtual survey. Similarly, the Green's function corresponding to the surface survey can be expressed as:

$$
D\left(\mathbf{x}_{r}, \mathbf{x}_{s}, t\right)=\int_{S_{D}} \mathrm{G}^{-}\left(\mathbf{x}_{r}, \mathbf{x}_{v r}, t\right) * \mathrm{G}^{-}\left(\mathbf{x}_{v r}, \mathbf{x}_{s}, t\right) d \mathbf{x}_{v r} .
$$

Substituting equation 1 into the above convolutional equations, the forward modeling for datuming (Guo and Alkhalifah, 2019a) is given by:

$\mathbf{F} u_{D}\left(\mathbf{x}, \mathbf{x}_{s}, t\right)=\iint u\left(\mathbf{x}_{v s}-\mathbf{h}, \mathbf{x}_{s}, t\right) * \mathrm{~V}\left(\mathbf{x}_{v s}-\mathbf{h}, \mathbf{x}_{v s}, t\right) \delta\left(\mathbf{x}-\mathbf{x}_{v s}\right) d \mathbf{h} d \mathbf{x}_{v s}$, where $u=\mathrm{G}^{+} * s$ and $u_{D}=D * s, s$ is a source function and $\mathbf{h}$ is the subsurface offset vector. We can see that the simulated wavefield from the datum is generated from the secondary source given by the convolution of the source wavefield with the redatumed virtual data.

Given the forward modeling operator shown in equation 4 , the redatumed virtual data $\mathrm{V}$ can be retrieved by minimizing the data misfits (Alkhalifah and $\mathrm{Wu}, 2016$; $\mathrm{Li}$ et al., 2019):

$$
\min _{\mathrm{v}} J=\frac{1}{2}\left\|\boldsymbol{\Gamma}\left(u+u_{D}\right)-d\right\|^{2},
$$

where, $d$ is the observed data and $\boldsymbol{\Gamma}$ is used to map the simulated wavefield to the receivers. The gradient of the objective function with respect to the redatumed data $\mathrm{V}$ is derived using the adjoint state method as follows:

$$
\frac{\partial J}{\partial \mathrm{V}}=-\sum_{s}\left\langle u\left(\mathbf{x}_{v s}, \mathbf{x}_{s}, t\right), u^{\dagger}\left(\mathbf{x}_{v s}+\mathbf{h}, \mathbf{x}_{s}, t\right\rangle_{t},\right.
$$

where, $u^{\dagger}$ is the backward propagating wavefield from the adjoint source at the receivers, which is for a least-square objective function given by the data misfits. We can compute the gradient for virtual data $\mathrm{V}$ by cross-correlating between the forward and backward propagating wavefields at the datum level, which also corresponds to the extended imaging condition for space- and time- extensions (Guo and Alkhalifah, 2019a). The inverted virtual dataset describes the scattering that occur only below the datum.

The redatuming process yields the virtual dataset as if the sources and receivers are located at the new datum level. With the redatumed data for the time-lapse survey in hand, we infer the time-lapse medium changes in the target zone below the datum from the redatumed data difference using a DDWI method (Denli and Huang, 2009).

\section{Examples}

We test the proposed approach on the Marmousi model, which is shown in Figure 1a and is viewed as the baseline model. We implant velocity changes into the baseline model to construct the monitor model. Figure $1 \mathrm{~b}$ shows the time-lapse changes of velocity in the target region which is the objective of the target-oriented inversion. We generate seismic data for the baseline and monitor model using 92 shots near the surface and 461 receivers at depth $0.2 \mathrm{~km}$. Starting from the initial model (Figure 2a), we use two frequency bands $3-12 \mathrm{~Hz}$ and $3-30 \mathrm{~Hz}$ sequentially to invert for the baseline model. The final inverted baseline model is shown in Figure $2 \mathrm{~b}$. To mitigate the effects of the overburden complexity, we set the datum at depth $2.18 \mathrm{~km}$ and apply the redatuming process to retrieve the scattering response for the underlying model below the datum for both the baseline and monitor data. Figure 3 a shows the redatumed virtual shot gathers for the baseline at the datum. Subtracting the baseline virtual data from the monitor virtual data, we obtain the time-lapse data difference for the virtual survey (Figure 3b). We first invert for the baseline model in the target region using the baseline virtual data and the inverted result is shown in Figure 4. The time-lapse velocity changes resolved from DDWI is shown in Figure 5 a. We can see that the velocity changes resolved by the proposed inversion algorithm are somewhat better than the corresponding time-lapse full-model inversion result (Figure 5b) where only the target zone is inverted. For a detailed comparison, we plot their vertical profiles at distance $6.5 \mathrm{~km}$ (Figure 5c). The target-oriented inversion captures the velocity changes at the right position with slight artifacts.

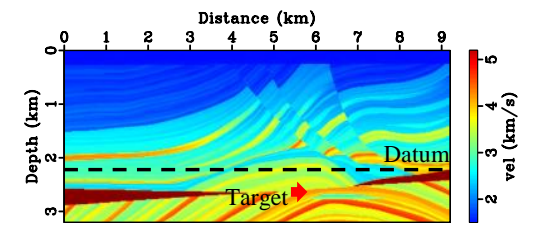

(a)

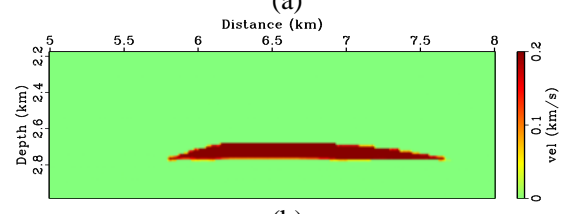

(b)

Figure 1: (a) The true baseline model and (b) the true time-lapse velocity changes in the target region. The dashed line shows the new datum. 


\section{Target-oriented time-lapse waveform inversion: Feasibility and robustness}

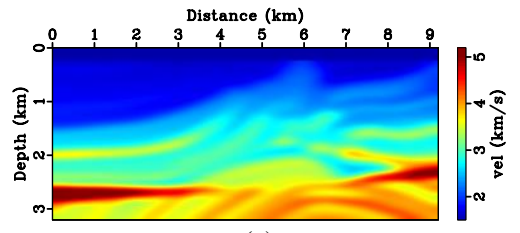

(a)

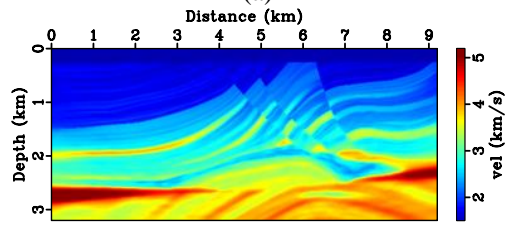

(b)

Figure 2: (a) The initial model and (b) the inverted baseline model using standard FWI.

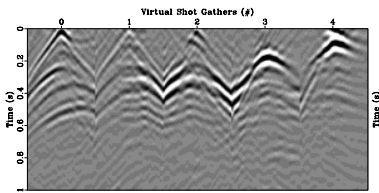

(a)

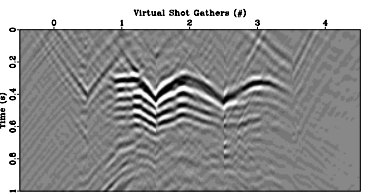

(b)
Figure 3: (a) The virtual shot gathers for baseline at the datum level and (b) the time-lapse data difference for the virtual survey.

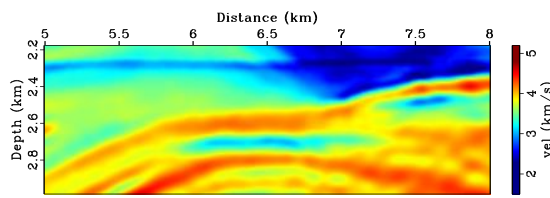

Figure 4: The inverted baseline model in the target region using the baseline virtual data shown in Figure $3 \mathrm{a}$.

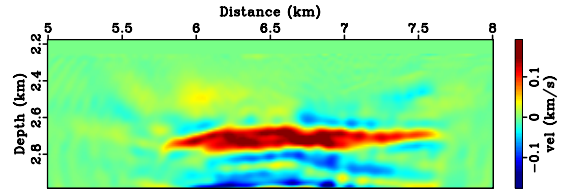

(a)

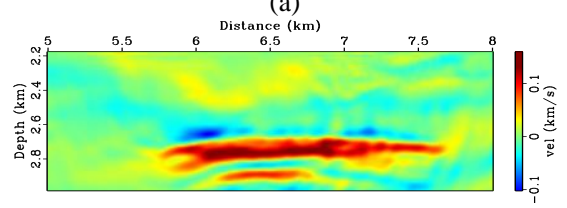

(b)

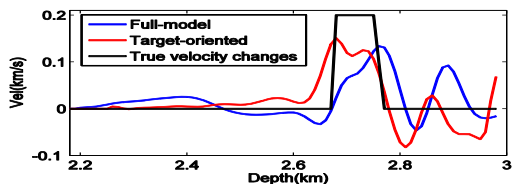

(c)

Figure 5: (a) The target-oriented and (b) full-model time-lapse inversion result. (c) The vertical profiles of the inverted and true velocity changes at $x=6.5 \mathrm{~km}$. Only the target zone is updated in the full-model inversion for fair comparison.

\section{Overburden model dependence}

In this section, we investigate how the quality of the overburden models affects the performance of the targetoriented time-lapse inversion. We use three overburden models those are respectively obtained from the initial model (Figure 2a), the inversion result using the frequency band $3-12 \mathrm{~Hz}$ and final inversion result (Figure $2 b$ ). Their vertical profiles are shown in Figure 6 . We perform the target-oriented time-lapse inversion workflow for each overburden model using the same number of iterations. The inverted velocity changes using the initial model and intermediate FWI result $(3-12 \mathrm{~Hz})$ are shown in Figure $7 \mathrm{a}$ and $7 \mathrm{~b}$, respectively. Note, the inverted changes corresponding to the final inversion result has been described in Figure 5a. For a detailed comparison, we plot their vertical profiles at distance $6.5 \mathrm{~km}$ in Figure $7 \mathrm{c}$.

When we use the initial overburden velocity which is far from the true velocity, the inversion method somewhat failed to capture the shape of the changes accurately, though it managed to at least identify the changes. However, the recovered changes are located deeper than their accurate position and also affected by some artifacts (Figure 7c). Using the better overburden models, the corresponding inversion results are closer to the true velocity changes because their overburden models describe the kinematics accurately. The comparison in the Figure 7 demonstrates the importance of the overburden velocity, but we still can obtain good results with a cheaper low frequency waveform inversion of the overburden.

\section{Nonrepeatability of acquisition survey}

Double-difference waveform inversion requires repeatability of seismic surveys (Yang et al. 2015). Small deviations between acquisition surveys may lead to obvious data differences that have nothing to do with the model changes. The redatuming technique renders the acquisition survey fixed at the datum level, thereby improving the repeatability. In this section, we investigate the robustness of the target-oriented inversion method in the presence of survey nonrepeatability.

We perturb the source or receiver positions slightly to simulate survey changes between the two surveys. Both the random and systematic perturbations are studied (Yang et al., 2015). For the random perturbation, the source and receiver positions are perturbed with one grid point shift to right or left randomly. Figure 8 a shows the corresponding inverted velocity changes from the data difference between virtual monitor and baseline data. For the systematic perturbations, all the sources in monitor survey are moved one grid point to the right and bottom. The velocity changes resolved by our inversion method are shown in Figure $8 \mathrm{~b}$. Compared with the inversion result for the fixed acquisition geometry, the inverted velocity changes are 


\section{Target-oriented time-lapse waveform inversion: Feasibility and robustness}

contaminated by more artifacts, but still located accurately with reasonable quality. We can see that the developed inversion method can deliver stable inversion results in the presence of small source and receiver location deviations.

\section{Random noise}

We would like to illustrate the robustness of the inversion algorithm in the presence of random noise. The robustness of inversion for the baseline model is not addressed here as our focus is the target, yet it can be improved by utilizing all available surveys to retrieve the average model (Kazei and Alkhalifah, 2017). Before inversion, we add a Gaussian white noise with different energy level to the observed baseline and monitor data set. We then run the inversion workflow using the contaminated data with a signal-to-noise (SNR) ratios of 15 and $0.5 \mathrm{~dB}$. The corresponding inversion results in Figure 9 show that the velocity changes can be identified from the background noise in seismic data. As more noise are added, the quality of the inversion result is deteriorates.

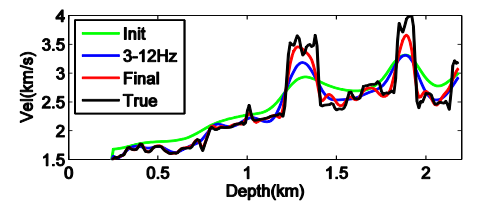

Figure 6: The vertical profiles of the overburden models at $x=6.5$ $\mathrm{km}$.
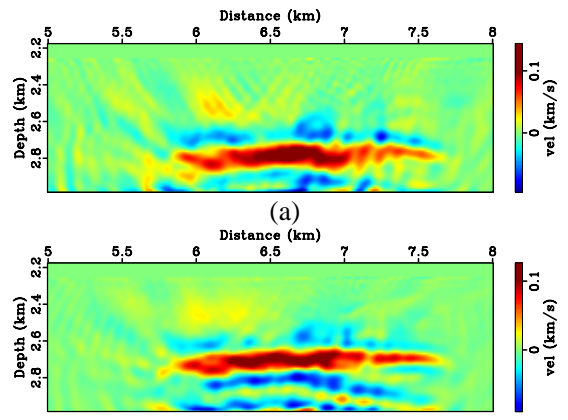

(b)

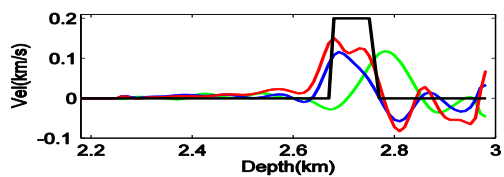

(c)

Figure 7: The recovered velocity changes using the overburden models corresponding to (a) the initial model and (b) the FWI inversion result using frequency band 3-12 Hz. (c) The vertical profiles of the inverted and true velocity changes at $\mathrm{x}=6.5 \mathrm{~km}$ (Green, blue and red lines refer to the inverted changes using the overburden models from initial, intermediate and final FWI results, respectively).

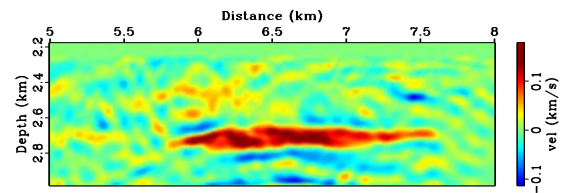

(a)

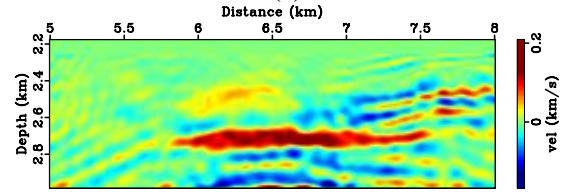

(b)

Figure 8: The inverted velocity changes in presence of nonrepeatable acquisition survey: (a) the source and receiver positions are randomly perturbed and (b) the source positions are systematically perturbed in monitor survey.

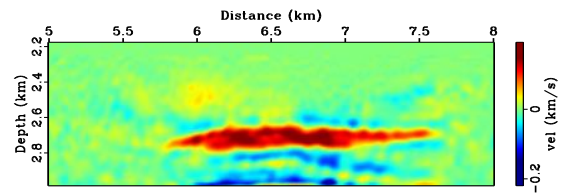

(a)

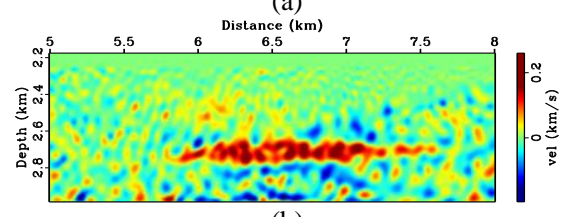

(b)

Figure 9: The time-lapse velocity changes obtained with noisy data with a SNR of (a) $15 \mathrm{~dB}$ and (b) $0.5 \mathrm{~dB}$.

\section{Conclusions}

We develop a target-oriented time-lapse waveform inversion scheme to retrieve property changes in the target zone effectively and efficiently. The virtual dataset for the target-oriented inversion is retrieved by redatuming the seismic data, usually recorded near the Earth's surface, to a new datum level. The numerical examples show that: (1) The target-oriented inversion delivers more accurate time-lapse velocity changes than the full-model inversion, even with a focus on the target zone;

(2) The velocity changes can be recovered clearly using an overburden model with accurate kinematics.

(3) The proposed inversion scheme shows strong robustness in presence of nonrepeatable acquisition survey and noise.

\section{Acknowledgements}

We would like to thank the Shaheen supercomputing Laboratory in KAUST for their computational support. We thank KAUST for its support and SWAG for collaborative environment. 


\section{REFERENCES}

Alkhalifah, T., and Z. Wu, 2016, The natural combination of full and image-based waveform inversion: Geophysical Prospecting, 64, 19-30, doi: https://doi.org/10.1111/1365-2478.12264.

Ayeni, G., and B. Biondi, 2010, Target-oriented joint least-squares migration/inversion of time-lapse seismic data sets: Geophysics, 75, no. 3, R61R73, doi: https://doi.org/10.1190/1.3427635.

Bakulin, A., and R. Calvert, 2006, The virtual source method: Theory and case study: Geophysics, 71, no. 4, SI139-SI150, doi: https://doi.org/10 $.1190 / 1.2216190$.

Borisov, D., and S.C. Singh, 2015, Three-dimensional elastic full waveform inversion in a marine environment using multicomponent ocean-bottom cables: a synthetic study: Geophysical Journal International, 201, no. 3, 1215-1234. doi: https://doi.org/10.1093/gjihttps://doi.org//ggv048.

Choi, Y., and T. Alkhalifah, 2011, Source-independent time-domain waveform inversion using convolved wavefields: Application to the encoded multisource waveform inversion: Geophysics, 76, no. 5, R125-R134, doi: https://doi.org/10.1190/geo2010-0210.1.

Denli, H., and L. Huang, 2009, Double-difference elastic waveform tomography in the time domain: 89th Annual International Meeting, SEG, Expanded Abstracts, 2302-2306.

Guo, Q., and T. Alkhalifah, 2019a, Datum-based waveform inversion using a subsurface-scattering imaging condition: Geophysics, 84, no. 4, S251S266, doi: https://doi.org/10.1190/geo2018-0615.1.

Guo, Q., and T. Alkhalifah, 2019b, Target-oriented inversion with least-squares waveform redatuming: 89th Annual International Meeting, SEG, Expanded Abstracts, 1521-1525.

Hicks, E., H. Hoeber, M. Houbiers, S.P. Lescoffit, A. Ratcliffe, and V. Vinje, 2016, Time-lapse full-waveform inversion as a reservoir-monitoring tool -A North Sea case study: The Leading Edge, 35, no. 10, 850-858, doi: https://doi.org/10.1190/tle35100850.1.

Kazei, V., and T. Alkhalifah, 2017, Centered differential waveform inversion with minimum support regularization: 79th Annual International Meeting, EAGE, Expanded Abstracts, 1-5.

Kazei, V., and T. Alkhalifah, 2018, Time-lapse waveform inversion regularized by spectral constraints and Sobolev space norm: 88th Annual International Meeting, SEG, Expanded Abstracts, 5487-5491.

Li, Y., and T. Alkhalifah, 2019, Matching-Filter Based Extended Full Waveform Inversion: 81st Annual International Meeting, EAGE, Expanded Abstracts, $1-5$.

Li, Y., T. Alkhalifah, and Z. Zhang, 2020, High-resolution regularized elastic full waveform inversion assisted by deep learning: 82nd Annual International Meeting, EAGE, Expanded Abstracts, 1-5.

Li, Y., Y. Choi, T. Alkhalifah, Z. Li, and K. Zhang, 2018, Full-waveform inversion using a nonlinearly smoothed wavefield: Geophysics, 83, no. 2, R117-R127, doi: https://doi.org/10.1190/geo2017-0312.1.

Li, Y., Q. Guo, Z. Li, and T. Alkhalifah, 2019, Elastic reflection waveform inversion with variable density: Geophysics 84, no. 4, R553-R567, doi: https://doi.org/10.1190/geo2017-0722.1.

Song, C., and T. Alkhalifah, 2020, Efficient wavefield inversion with outer iterations and total variation constraint: IEEE Transactions on Geoscience and Remote Sensing.

Tarantola, A., 1984, Inversion of seismic reflection data in the acoustic approximation: Geophysics, 49, 1259-1266. doi: https://doi.org/10.1190/1 .1441754 .

van Manen, D.-J., J. O. A. Robertsson, and A. Curtis, 2007, Exact wave field simulation for finite-volume scattering problems: The Journal of the Acoustical Society of America, 122, EL115-EL121, doi: https://doi.org/10.1121/1.2771371.

Vasconcelos, I., M. Ravasi, and J. van der Neut, 2017, Subsurface-domain, interferometric objective functions for target-oriented waveform inversion: Geophysics, 82, no. 4, A37-A41, doi: https://doi.org/10.1190/geo2016-0608.1.

Vigh, D., K. Jiao, D. Watts, and D. Sun, 2014, Elastic full-waveform inversion application using multicomponent measurements of seismic data collection: Geophysics, 79, R63-R77, doi: https://doi.org/10.1190/geo2013-0055.1.

Wapenaar, K., and J. van der Neut, 2010, A representation for green function retrieval by multidimensional deconvolution: Journal Acoustical Society of America, 128, EL366-EL371, doi: https://doi.org/10.1121/1.3509797.

Yang, D., M. Meadows, P. Inderwiesen, J. Landa, A. Malcolm, and M. Fehler, 2015, Double-difference waveform inversion: Feasibility and robustness study with pressure data: Geophysics, 80, no. 6, M129-M141, doi: https://doi.org/10.1190/geo2014-0489.1.

Yang, D., Y. Zheng, M. Fehler, and A. Malcolm, 2012, Target-oriented time-lapse waveform inversion using virtual survey: 82nd Annual International Meeting, SEG, Expanded Abstracts, 1-5.

Yuan, S., N. Fuji, S. Singh, and D. Borisov, 2017, Localized time-lapse elastic waveform inversion using wavefield injection and extrapolation: 2-D parametric studies: Geophysical Journal International, 209, no. 3, 1699-1717, doi: https://doi.org/10.1093/gjihttps://doi.org//ggx118. 